\title{
Power-balanced modeling of nonlinear coils and transformers for audio circuits
}

\author{
JUDY NAJNUDEL, THOMAS HÉLIE, DAVID ROZE AND RÉMY MÜLLER \\ (judy.najnudel@ircam.fr)（thomas.helie@ircam.fr) (david.roze@ircam.fr) (remy.muller@ircam.fr) \\ S3AM team, STMS laboratory \\ IRCAM - CNRS - SU \\ Paris, France
}

\begin{abstract}
This paper is concerned with the modeling of ferromagnetic coils with audio applications in mind. The proposed approach derives a macroscopic, energy-based formulation from statistical physics. This choice allows for thermodynamic variables to be explicitly taken into account. As a consequence, macroscopic features such as saturation and hysteresis arise directly. As the proposed model is expressed through a port-Hamiltonian formulation, power balance and passivity are guaranteed. Moreover, the model may be straightforwardly connected to other multi-physical components, and included into more complex systems. The proposed model is compared to measurements on a real ferromagnetic coil. Simulations of a passive band-pass filter and a transformer built around the model are presented as an illustration.
\end{abstract}

\section{INTRODUCTION}

Coils and transformers built around ferromagnetic cores are largely present in audio circuits, from emblematic effect pedals to amplifiers and loudspeakers. Ferromagnetic materials are sought for their high inductance, which increases the coil quality factor. On the other hand, these materials exhibit non-linear characteristics such as saturation and hysteresis, which may cause audible distortion and power loss. Understanding ferromagnetic materials is therefore necessary in order to accurately predict a coil's behavior, so that one may carefully avoid - or exploit distortion when designing or simulating circuits.

Ferromagnetism is a long-enduring research field, and several empirical macroscopic models already exist in the literature. One of the most widely used in the audio community is the Jiles-Atherton model [1, 2, 3], which is built around a differential equation involving a saturation curve and a friction term. However, some concern has been expressed regarding its physical interpretation [4], and subsequent accuracy issues in simulations. Another popular model is the Gyrator-Capacitor [5, 6, 7], whose strength resides in its simplicity. Indeed, it essentially consists in a Gyrator-Capacitor representation where the capacitor has a polynomial law. Other recent models based on fractional derivatives [8] have proved particularly accurate, but like the Jiles-Atherton or GC models previously mentioned, their parameters are not related to actual physical quantities. Moreover, neither of these models takes explicitly into account the significant role of temperature in the shape of the hysteresis curve. Yet temperature may vary in circuits, especially after an extended use; therefore its influence should not be entirely neglected. On the other hand, models explicitly built on energetic considerations, such as variational models [9], rely on costly finite-element methods, making real-time use difficult. Similarly, the Preisach model [10, 11] thoroughly captures the phenomenology involved in ferromagnetism, including thermodynamics; but it is obviously too complex for audio applications. Therefore to our present knowledge, a model both physicallybased (allowing refined and realistic simulations in a wide range of contexts) and suitable for audio applications does not seem to exist.

In this paper, we propose a non-linear model of ferromagnetic coil that is physically-based, passive (no hidden sources of energy), modular (allowing electric and thermal connections) and with a reduced complexity (only 3 state variables and 5 parameters).

First, we derive a core macroscopic model from classic statistical physics results, with a special care brought to the choice of state variables for thermodynamic consistency and modularity. Then, we build a ferromagnetic coil model connecting a core and a coil. It ensues that typical characteristics (hysteresis or its absence thereof) naturally arise from the interaction between the coil and the core, in association with features intrinsically present in our core model (meta-stability or stability). Moreover, as the model dy- 
namics is expressed through a Port-Hamiltonian Systems (PHS) formulation [12, 13], the power balance is structurally fulfilled. This formulation also makes the connection to other components straightforward, so that including the model in more complex systems poses no difficulty.

This ferromagnetic coil model is then used to simulate two circuits: a passive band-pass filter, and a transformer. The circuits are also modeled as PHS, and the simulations are based on numerical methods [14] that preserve the power balance in the discrete-time domain.

The paper is structured as follows: in section 11, the PortHamiltonian formalism is briefly presented. In section 2 the core macroscopic model is constructed from statistical physics and thermodynamics. Section 3 addresses the connection between the core and the coil, and describes the resulting complete model. In section 4, the model is assessed against measurements on a Fasel inductor. Finally, simulations of chosen audio circuits are presented in section 5, before providing elements of discussion and work perspectives in section 6

\section{PORT-HAMILTONIAN SYSTEMS: DIFFERENTIAL-ALGEBRAIC FORMULATION}

All subsequent modeling relies on Port-Hamiltonian systems [15, 13], under a differential-algebraic formulation [14]. This formulation allows the representation of a dynamical system as a network of:

1. storage components of state $\boldsymbol{x}$ and energy $E(\boldsymbol{x})$;

2. dissipative components described by an effort law $\boldsymbol{w} \mapsto$ $z(\boldsymbol{w})$, such as the dissipated power $P_{\mathrm{diss}}=z(\boldsymbol{w})^{\top} \boldsymbol{w}$ is non-negative for all flows $\boldsymbol{w}$;

3. connection ports conveying the outgoing power $P_{\mathrm{ext}}=$ $\boldsymbol{u}^{\top} \boldsymbol{y}$ where $\boldsymbol{u}$ are inputs and $\boldsymbol{y}$ are outputs.

The flows $\boldsymbol{f}$ and efforts $\boldsymbol{e}$ of all the components are coupled through a skew-symmetric interconnection matrix $\boldsymbol{J}=-\boldsymbol{J}^{\top}$ :

$$
\underbrace{\left[\begin{array}{c}
\dot{x} \\
\boldsymbol{w} \\
\boldsymbol{y}
\end{array}\right]}_{f}=\underbrace{\left[\begin{array}{c}
\nabla E(\boldsymbol{x}) \\
z(\boldsymbol{w}) \\
\boldsymbol{u}
\end{array}\right]}_{\boldsymbol{e}} .
$$

Here, flows can either be currents (e.g. for capacitors) or voltages (e.g. for inductors), and vice versa for efforts. Such systems satisfy the power balance $P_{\text {stored }}+P_{\text {diss }}+P_{\text {ext }}=0$ where $P_{\text {stored }}=\nabla E(\boldsymbol{x})^{\top} \dot{\boldsymbol{x}}$ denotes the stored power. Indeed, $P_{\text {stored }}+P_{\text {diss }}+P_{\text {ext }}=e^{\top} \boldsymbol{f}=$ $\boldsymbol{e}^{\top} \boldsymbol{J} \boldsymbol{e}$ is zero since $\boldsymbol{e}^{\top} \boldsymbol{J} \boldsymbol{e}=\left(\boldsymbol{e}^{\top} \boldsymbol{J} \boldsymbol{e}\right)^{\top}=-\left(\boldsymbol{e}^{\top} \boldsymbol{J} \boldsymbol{e}\right)$ due to the skew-symmetry of $\boldsymbol{J}$.

All models herein will be formulated as (1).

\section{FERROMAGNETIC CORE MODELING}

In this section, a macroscopic model of the ferromagnetic core is derived from a microscopic representation, known in the literature as the Ising model [16, 17, 18]. The

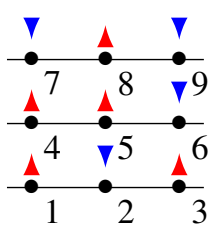

Figure 1: Possible micro-state $\boldsymbol{m}$ of a core with $N=9$ moments.

change of scale is performed within the statistical physics framework. As the use of statistical physics leads to the emergence of thermodynamic variables, the latter are taken into account explicitly in the modeling. Consequently, the chosen macroscopic state and its corresponding internal energy allow the connection of the core to both electromagnetic ports and thermal ports. These ports, through the PHS formalism, control the influence of an external magnetic field on one hand (ultimately responsible for the presence of hysteresis, as will be shown), and the influence of phase transitions on the other hand (responsible for the amount of hysteresis) on the core state and its subsequent dynamics.

Understanding the Ising model at a quantum level is not the object of this paper. Here we only give necessary elements in order to derive a macroscopic model. Similarly, we do not propose new results on statistical physics but use it in a standard way as a mean to an end. Therefore, we present the main concepts without detailing all intermediate steps. Readers who wish to deepen their knowledge on the subject may refer to [19, 20, 21].

\subsection{Ising model}

In the Ising model, a ferromagnetic core is represented as a set of $N$ adimensional magnetic moments, interacting with one another. A possible state $\boldsymbol{m}$ of the core (called micro-state in the following) is therefore a particular configuration of these moments: $\boldsymbol{m} \in \mathbb{M}=$ $\{-1,1\}^{N}$. Figure 1 shows a possible micro-state $\boldsymbol{m}=$ $[1,-1,1,1,1,-1,-1,1,-1]^{\top}$ for $N=9$.

The interactions between the micro-state moments are expressed through the Heisenberg hamiltonian $\mathscr{H}(\boldsymbol{m})$ :

$$
\mathscr{H}(\boldsymbol{m})=-\frac{1}{2} \boldsymbol{m}^{\top} \mathscr{J}_{\mathrm{ex}} \boldsymbol{m},
$$

where each coefficient $\mathscr{J}_{\mathrm{ex}_{i, j}}$ is the exchange energy [22] between moment $i$ and moment $j$. Assuming isotropic interactions affecting nearest neighbours only, this exchange energy simplifies to:

$$
\mathscr{J}_{\mathrm{ex}_{i, j}}= \begin{cases}J & i, j \text { nearest neighbours }, i \neq j, \\ 0 & \text { else },\end{cases}
$$

where $J$ is a constant energy characterizing the material.

For ferromagnetic materials, $J$ is positive; consequently, the Heisenberg hamiltonian minimizes itself for configurations in which moments align with one another. 


\subsection{Statistical physics}

The micro-state of the core may fluctuate randomly over time, without affecting its overall macroscopic properties: indeed, several micro-states yield the same hamiltonian. Therefore, the specific micro-state of the core at any given time cannot be known from macroscopic observations. Thus, we rely on a probabilistic description of the core (refered to as the system in the following) to predict its macroscopic behavior.

To this end, we consider the canonical ensemble, which is the thermodynamic ensemble (i.e., a time-invariant probability space) of interest for a system under the following set of assumptions hyp:

1. The system is closed (constant number of atoms $N$ ).

2. The system is isochoric (constant volume $V$ ).

3. The system may exchange energy with the exterior (its energy fluctuates over time).

4. The exterior is much larger than the system and behaves like a thermostat $\left(T_{\text {core }}=T_{\text {ext }}=T\right)$.

The first two assumptions are taken into account in the expression of the hamiltonian in Eq. (2). The fourth assumption constrains the energy exchanges between the system and the exterior.

With this set hyp, we look for the micro-states probability distribution for the canonical ensemble at thermodynamic equilibrium, denoted $p_{\text {hyp }}^{\star}$. This distribution derives from a fundamental principle of statistical physics: the statistical entropy maximization at thermodynamic equilibrium.

Derivation. Given a probability distribution $p$ of microstates, the statistical entropy measures the amount of information required to know the exact micro-state of the system. It is defined as:

$$
\mathrm{S}: p \longmapsto-k_{b} \sum_{\boldsymbol{m} \in \mathbb{M}} p(\boldsymbol{m}) \ln p(\boldsymbol{m})
$$

where $k_{b}=1.38 \times 10^{-23} \mathrm{~J} . \mathrm{K}^{-1}$ is the Boltzmann constant. Indeed, for a distribution mapping some micro-state $\boldsymbol{m}_{0}$ to 1 and the others to 0 , the system is entirely known to be in the state $\boldsymbol{m}_{0}$. According to Eq. (4), this distribution would yield a zero entropy (the definition of $\mathrm{S}$ can be extended to 0 since $\lim _{x \rightarrow 0} x \ln x=0$ ). Conversely, an equiprobable distribution between all micro-states maximizes the lack of information on the system. From Eq. (4), this distribution also maximizes the entropy.

When a system reaches thermodynamic equilibrium, it stops evolving. At this point the only information available is the information corresponding to our assumptions on the system. Since this information is minimal (any less information would characterize a different system), the system entropy should be maximal. Moreover, at equilibrium, the third assumption in hyp actually translates into the ergodic hypothesis. This hypothesis stipulates that at equilibrium, the internal energy defined as the mean energy over time $\bar{E}$, also coincides with the expectation of the hamiltonians of all possible micro-states:

$$
\bar{E}=\mathbb{E}[\mathscr{H}]:=\sum_{\boldsymbol{m} \in \mathbb{M}} p^{\star}{ }_{\text {hyp }}(\boldsymbol{m}) \mathscr{H}(\boldsymbol{m}) .
$$

Therefore for the canonical ensemble, the entropy maximization at equilibrium can be written as:

$$
\begin{aligned}
p_{\text {hyp }}^{\star}= & \underset{p}{\arg \max } \mathrm{S}(p) \\
& \text { subject to } \bar{E}=\mathbb{E}[\mathscr{H}] .
\end{aligned}
$$

Solving Eq. (6) (usually with Lagrange multipliers) yields the well-known Boltzmann distribution for the canonical ensemble:

$$
\begin{aligned}
p^{\star}{ }_{\text {hyp }}(\boldsymbol{m}) & =\frac{\exp \left(-\frac{\mathscr{H}(\boldsymbol{m})}{k_{b} T}\right)}{\mathscr{Z}(T)}, \\
\mathscr{Z}(T) & =\sum_{\boldsymbol{m} \in \mathbb{M}} \exp \left(-\frac{\mathscr{H}(\boldsymbol{m})}{k_{b} T}\right) .
\end{aligned}
$$

The dependency to the temperature $T$ is directly related to the constraint on $\bar{E}$. Indeed, it can be shown that the associated Lagrange multiplier $\lambda$, which appears in $p_{\text {hyp }}^{\star}$ during the derivation, is $\lambda=-\partial \mathrm{S} / \partial \bar{E}=-1 / T_{\text {core }}=-1 / T$ (see e.g. [23] for a detailed derivation). The denominator $\mathscr{Z}(T)$ defines the partition function of the system and acts as a normalization factor. From Eq. (7), it is clear that at low temperatures, micro-states with a lower hamiltonian (i.e., whose moments are aligned with each other) are favored, whereas at high temperatures, all micro-states tend to be equiprobable. It follows that the higher the temperature, the higher the entropy, but the Boltzmann distribution is nonetheless the distribution maximizing the entropy at a given temperature.

Finally, reinjecting Eq. (7) in Eq. (4) and Eq. (5) yields the thermodynamic entropy and the internal energy as functions of the temperature:

$$
\begin{gathered}
S=\mathrm{S}\left(p_{\text {hyp }}^{\star}\right)=\mathscr{S}(T):=\frac{\partial}{\partial T}\left(k_{b} T \ln \mathscr{Z}\right)(T), \\
\bar{E}=\mathscr{E}(T):=k_{b} T^{2} \frac{\partial \ln \mathscr{Z}}{\partial T}(T)
\end{gathered}
$$

(see e.g. [24] for detailed proof).

\subsection{Core macroscopic state and energy}

The classic mean-field Ising model computes an approximation of the free energy, which corresponds to the amount of energy convertible into work, at constant temperature. This mean-field free energy depends on a macroscopic, scalar order parameter $m \in[-1,1]$. This order parameter can be understood as a "mean magnetic moment". It is \pm 1 when moments tend to align for all micro-states, 0 if there is no alignment tendency at all. The free energy, in its classic formulation, also depends on the external magnetic field (see e.g. [25] for a complete derivation).

Here, we also rely on a mean-field approximation, but choose to express the internal energy as a function of the (extensive) entropy $S$ and another extensive variable, the total magnetic flux $B_{V}$. This way, in (1), the flow $\dot{\boldsymbol{x}}_{\text {core }}$ accounts for the time variation of extensive quantities (to bal- 
ance with quantities external to the core, or equilibriumestablishing) and, concomitantly, the effort accounts for intensive quantities (shared with the exterior at the core interface, or equilibrium-determining). Thus, the externality of the thermostat and magnetic field is made explicit. The core macroscopic state can only change if the core is in contact with the exterior (or another component) through connection ports.

Assuming the core has periodic boundaries (a toric geometry for instance), and reinjecting the mean-field partition function of a core isolated from any magnetic field (see [18] for a complete expression) in Eq. (9), the mean-field internal energy is found to be:

$$
\bar{E} \approx \mathscr{E}_{M F}(m, T)=E_{0}\left(\frac{m^{2}}{2}-m \tanh \left(\frac{m T_{c}}{T}\right)\right) .
$$

In Eq. (10), the energy $E_{0}=N J q$, with $q$ the (constant) number of nearest neighbours of each moment, relates to the minimal possible energy of the core: $\mathscr{E}_{M F}( \pm 1,0)=$ $-E_{0} / 2$.

A similar operation in Eq. (8) gives the mean-field thermodynamic entropy:

$$
\begin{aligned}
& S \approx \mathscr{S}_{M F}(m, T)=S_{0} f\left(\frac{m T_{c}}{T}\right), \\
& \quad f(\chi)=\ln (2 \cosh (\chi))-\chi \tanh (\chi), \chi \in \mathbb{R} .
\end{aligned}
$$

In Eq. (11), the entropy $S_{0}=N k_{b}$ relates to the maximal possible entropy of the core: $\mathscr{S}_{M F}(0,+\infty)=S_{0} \ln (2)$.

The ratio $E_{0} / S_{0}:=T_{c}$ gives the critical temperature, above which the core behavior becomes paramagnetic.

Simultaneously, we introduce the (extensive) core total magnetic flux $B_{V_{\text {core }}}$, defined as:

$$
B_{V_{\text {core }}}=m \mu_{0} M_{s} V
$$

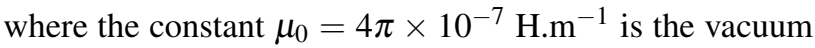
magnetic permeability, the quantity $M_{s}$ is the core saturation magnetization, and $V$ is the core volume. The term $\mu_{0} M_{s} V$, corresponding to the core saturation total magnetic flux, is denoted $B_{V_{s}}$ in the following for simplicity.

Finally, the core macroscopic state is chosen to be expressed as:

$$
\boldsymbol{x}_{\text {core }}=\left[B_{V_{\text {core }}}, S\right]^{\top} \text {. }
$$

Noting that $f$ is smooth, even on $\mathbb{R}$ and strictly monotonic ${ }^{1}$ (so invertible) on $\mathbb{R}^{+}$, we obtain the expression of the core internal energy:

$$
E_{\text {core }}\left(\boldsymbol{x}_{\text {core }}\right)=E_{0}\left(\frac{1}{2}\left(\frac{B_{V_{\text {core }}}}{B_{V_{\mathrm{s}}}}\right)^{2}-\left|\frac{B_{V_{\text {core }}}}{B_{V_{\mathrm{s}}}}\right| g(S)\right),
$$

where $g(S)=\tanh \left(f^{-1}\left(\frac{S}{S_{0}}\right)\right), S \in \mathbb{R}^{+}$. The effort is:

$$
\begin{aligned}
\nabla E_{\text {core }}\left(\boldsymbol{x}_{\text {core }}\right) & =\left[\frac{\partial E_{\text {core }}}{\partial B_{V_{\text {core }}}}\left(\boldsymbol{x}_{\text {core }}\right), \frac{\partial E_{\text {core }}}{\partial S}\left(\boldsymbol{x}_{\text {core }}\right)\right]^{\top} \\
& :=\left[H_{\text {core }}, T_{\text {core }}\right]^{\top}
\end{aligned}
$$

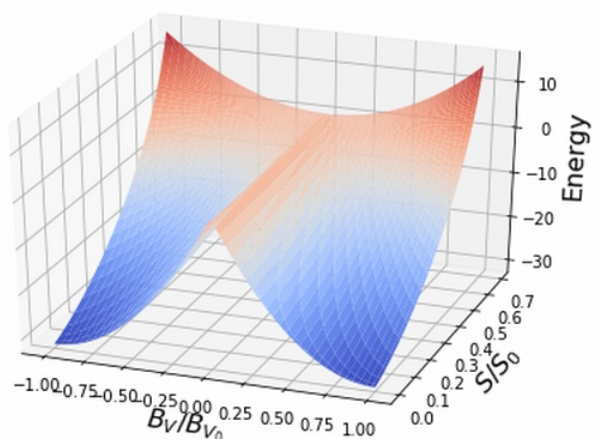

Figure 2: Energy function of the core.

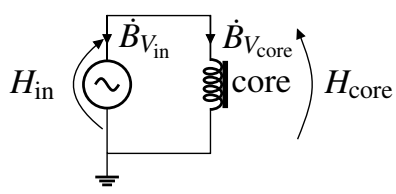

(a) Flow-controlled core.

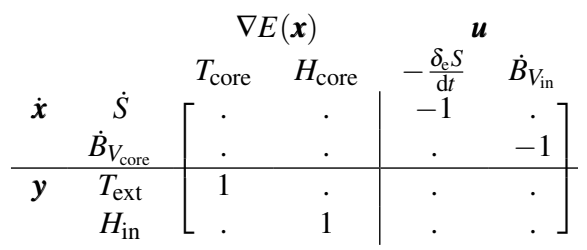

(b) Corresponding PHS. Dots represent zeros.

Figure 3: Equivalent circuit and PHS for the flowcontrolled core.

where $H_{\text {core }}$ and $T_{\text {core }}$ denote the core internal magnetic field and the core temperature, respectively. The quantity $T_{\text {core }}$ defined in Eq. (15) coincides exactly with the temperature $T$ used in Eq. (7), thus ensuring the thermodynamic consistency of the model (proof in Appendix A.1).

Figure 2 shows how as the entropy $S$ increases, the core goes from two ordered (aligned moments) meta-stable equilibrium states to one non-ordered (no alignment) stable equilibrium state: it exhibits a phase transition (from ferromagnetic to paramagnetic). Note that all non-linearities of the core are intrinsically encoded into its energy. Therefore any PHS comprising a core will exhibit a non-linear behavior, regardless of its interconnection matrix.

\subsection{Flow-controlled conservative PHS}

We suppose that the core is flow-controlled. The outgoing total magnetic flux and incoming entropy variation from the exterior are denoted $B_{V_{\text {in }}}$ and $\delta_{\mathrm{e}} S$, respectively ${ }^{2}$ Figure 3 a shows the equivalent circuit. Kirchhoff's laws in receptor convention, as well as Eq. (14), yield the PHS formulation in Fig. 3b.

\footnotetext{
${ }^{1}$ Indeed, $f^{\prime}(\chi)=-\chi / \cosh ^{2} \chi \leq 0 \forall \chi \in \mathbb{R}^{+}$.
}

\footnotetext{
${ }^{2}$ The symbol $\delta$ stands for an inexact differential [21].
} 


\subsection{Effort-controlled dissipative PHS}

We suppose now that the core is effort-controlled. In this case, the exterior magnetic field $H_{\text {in }}$ constrains the core magnetic field:

$$
H_{\text {core }}=H_{\text {in }} .
$$

Below the critical temperature, this constraint causes jumps between meta-stable states (called Barkhausen jumps [26], Fig. 4a). The subsequent relaxation is responsible for hysteresis, similarly to a relaxation oscillator [27]. In real ferromagnetic cores however, domain structure and non-homogeneities [28] generate an energy function with not two but multiple local minima. Therefore, on a macroscopic scale, the trajectory followed by the magnetic flux during jumps is damped (Fig. 4b). The damping is modeled with a linear magnetic resistor $r_{\text {core }}$ connected in series with the core.

Above the critical temperature, there is no meta-stability, and the remaining hysteresis is mainly due to eddy currents.

As the difference of energy before and after a jump is entirely dissipated as heat [26], the Barkhausen jumps are also responsible for the variation of entropy creation $\delta_{i} S$ in the core. The associated thermal power $T_{\text {core }} \delta_{i} S / \mathrm{d} t$ is equal to the magnetic power dissipated through $r_{\text {core }}$, so that

$$
r_{\text {core }} H_{r_{\text {core }}}^{2}-T_{\text {core }} \frac{\delta_{i} S}{\mathrm{~d} t}=0
$$

We deduce the dissipative flow and effort:

$$
\begin{aligned}
\boldsymbol{w} & =\left[-T_{\text {core }}, H_{r_{\text {core }}}\right]^{\top} \\
z(\boldsymbol{w}) & =\left[\frac{r_{\text {core }} H_{r_{\text {core }}}^{2}}{T_{\text {core }}}, r_{\text {core }} H_{r_{\text {core }}}\right]^{\top}=\left[\frac{\delta_{i} S}{\mathrm{~d} t}, \dot{B}_{V_{r_{\text {core }}}}\right]^{\top} .
\end{aligned}
$$

Finally, the second law of thermodynamics [21] states:

$$
\dot{S}=\frac{\delta_{i} S}{\mathrm{~d} t}+\frac{\delta_{e} S}{\mathrm{~d} t} .
$$

Figure 5 a shows the equivalent circuit. Kirchhoff's laws in receptor convention, as well as Eqs. (14)-(18)-(19), yield the PHS formulation in Fig. $5 b$

\section{CONNECTION TO COIL}

\subsection{Coil model}

The coil is a (considered linear here) component which can be described either in the electrical domain or the magnetic domain.

In the electrical domain, its state is the magnetic flux linkage $\Phi_{\text {coil }}$ and its energy is:

$$
E_{\text {coil }}^{\text {elec }}\left(\Phi_{\text {coil }}\right)=\frac{\Phi_{\text {coil }}^{2}}{2 L}
$$

with $L$ the coil inductance. Its energy derivative w.r.t. $\Phi_{\text {coil }}$ is the coil current $i_{\text {coil }}=\Phi_{\text {coil }} / L$. is:

In the magnetic domain, its state is $B_{V_{\text {coil }}}$ and its energy

$$
E_{\text {coil }}^{\text {mag }}\left(B_{V_{\text {coil }}}\right)=\frac{B_{V_{\text {coil }}}^{2}}{2 \mu_{0} V_{\text {coil }}}
$$

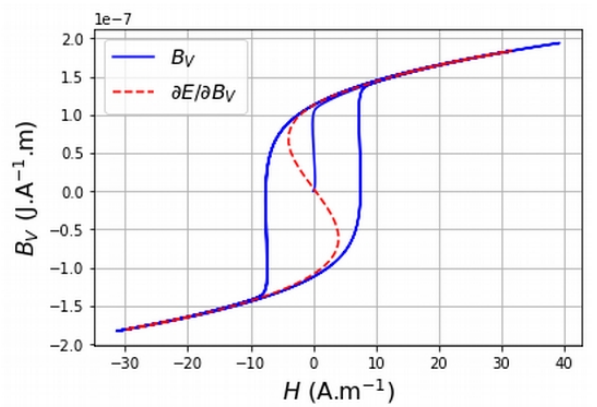

(a) Barkhausen jumps without damping.

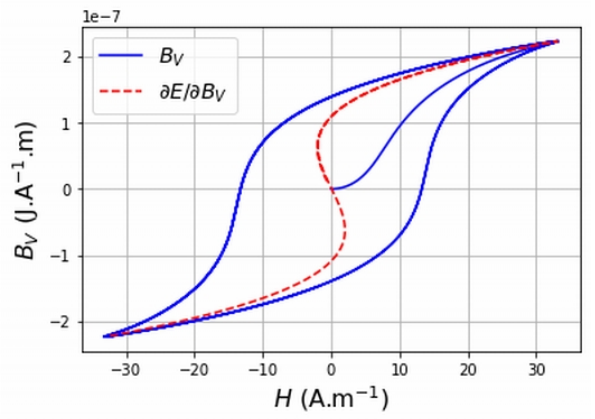

(b) Barkhausen jumps with damping.

Figure 4: Barkhausen jumps and resulting hysteresis during a cycle, with and without damping. The red curve is the theoretical core internal magnetic field, the blue curve is the real trajectory followed by the core total magnetic flux constrained by an external magnetic field.

with $V_{\text {coil }}$ the coil volume. Its energy derivative w.r.t. $B_{V_{\text {coil }}}$ is the coil magnetic field $H_{\text {coil }}=B_{V_{\text {coil }}} /\left(\mu_{0} V_{\text {coil }}\right)$.

Obviously, $E_{\text {coil }}^{\text {elec }}\left(\Phi_{\text {coil }}\right)=E_{\text {coil }}^{\mathrm{mag}}\left(B_{V_{\text {coil }}}\right)$. Moreover, denoting $n$ the number of turns and $\ell$ the length of the coil, the coil flows and efforts in both domains are related through:

$$
\left[\begin{array}{c}
H_{\text {coil }} \\
\dot{\Phi}_{\text {coil }}
\end{array}\right]=\left[\begin{array}{cc}
0 & \frac{n}{\ell} \\
\frac{n}{\ell} & 0
\end{array}\right]\left[\begin{array}{c}
\dot{B}_{V_{\text {coil }}} \\
i_{\text {coil }}
\end{array}\right] .
$$

The coil can therefore be seen as an interface between the electric and magnetic domains. This leads to the equivalent quadripole representations shown in Fig. 6. In the following, the coil will therefore be represented as such a quadripole, to account for its double nature.

\subsection{Coupling between the core and the coil}

A ferromagnetic coil is formed when a core and a coil are connected (in series in the electrical domain, in parallel in the magnetic domain). As such, the magnetic flux of the coupled system is the sum of the magnetic fluxes:

$$
B_{V_{\text {coupled }}}=B_{V_{\text {coil }}}+B_{V_{\text {core }}},
$$

and they share their magnetic field:

$$
H_{\text {coupled }}=H_{\text {core }}=H_{\text {coil }} .
$$

Therefore, the coil acts as an effort-controller for the core, and the core dynamics is that described in section 2.5 


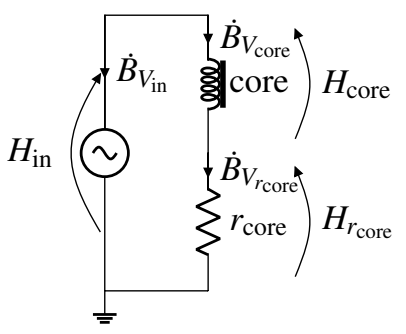

(a) Effort-controlled core with dissipation.

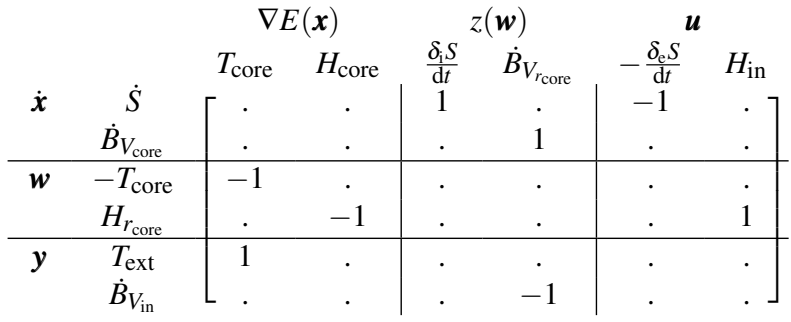

(b) Corresponding PHS. Dots represent zeros.

Figure 5: Equivalent circuit and PHS for the effort-controlled core.
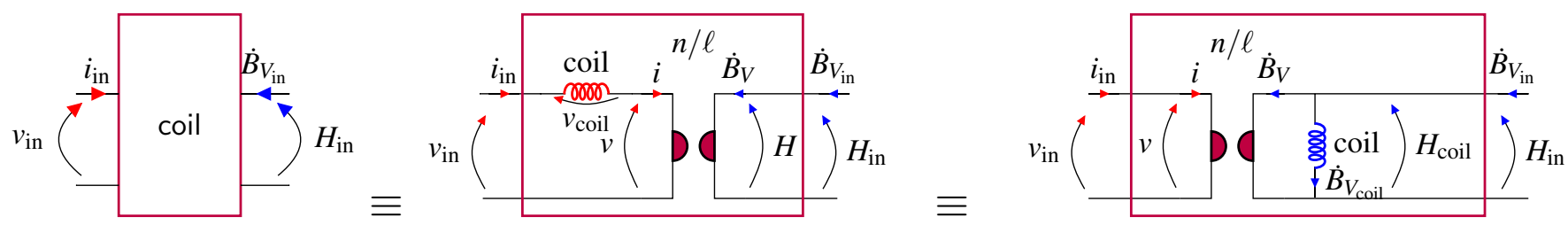

Figure 6: Coil equivalent representations as interfaces between electric (red) and magnetic (blue) domains.

\subsection{Complete ferromagnetic coil model}

The losses due to Joule heating in the coil are modeled with a linear resistor $r_{\text {coil }}$ in series (in the electrical domain) with the coil, so that for the ferromagnetic coil, the dissipative flows and efforts are:

$$
\begin{aligned}
\boldsymbol{w} & =\left[-T_{\text {core }}, H_{r_{\text {core }}}, i_{r_{\text {coil }}}\right]^{\top} \\
z(\boldsymbol{w}) & =\left[\frac{r_{\text {core }} H_{r_{\text {core }}}^{2}}{T_{\text {core }}}, r_{\text {core }} H_{r_{\text {core }}}, r_{\text {coil }} i_{r_{\text {coil }}}\right]^{\top} \\
& =\left[\frac{\delta_{i} S}{\mathrm{~d} t}, \dot{B}_{V_{r_{\text {core }}}}, v_{r_{\text {coil }}}\right]^{\top} .
\end{aligned}
$$

The ferromagnetic coil equivalent circuit is shown on Fig. 7a Kirchhoff's laws on the equivalent circuit, as well as Eqs. (14)-(19)-(21)-(22)-(25), allow the PHS formulation on Fig. $7 b$

\subsection{Isothermal transformations}

In the case of interactions with a ferromagnetic coil at constant temperature, one may use an alternate formulation. The entropy is removed from the state, which becomes:

$$
\boldsymbol{x}=\left[B_{V_{\text {core }}}, B_{V_{\text {coil }}}\right]^{\top} .
$$

The core internal energy becomes the free energy:

$E_{\text {core }}^{\text {free }}\left(B_{V_{\text {core }}}\right)=E_{0}\left(\frac{1}{2}\left(\frac{B_{V_{\text {core }}}}{B_{V_{\mathrm{s}}}}\right)^{2}-\theta \ln \cosh \left(\frac{B_{V_{\text {core }}}}{B_{V_{s}} \theta}\right)\right)$,

where $\theta=T / T_{c}$ is now a parameter of the model. The dissipative flow becomes:

$$
\boldsymbol{w}=\left[H_{r_{\text {core }}}, i_{r_{\text {coil }}}\right]^{\top},
$$

and the only input is $v_{\text {in }}$. The PHS of the ferromagnetic coil is otherwise unchanged.
Nonetheless, the applications presented in the next section will rely on the formulation given in section 3.3 , as it is more general.

\section{COMPARISON TO MEASUREMENTS}

To assess the accuracy of the model, measurements are performed on a Fasel Red inductor. The inductor is connected in series with a resistor $R=100 \Omega$ and driven with a sinusoidal voltage source (Fig. 8a). The voltage across the inductor $v_{\text {out }}$ is measured, and the inductor magnetic flux linkage is obtained through the relation $\Phi_{\mathrm{L}}(t)=$ $\int_{0}^{t} v_{\text {out }}(\tau) \mathrm{d} \tau$. The current $i_{\mathrm{L}}$ is obtained through the relation $i_{\mathrm{L}}=\left(v_{\text {in }}-v_{\text {out }}\right) / R$. Assuming that $T=303 \mathrm{~K}, \ell=$ $0.016 \mathrm{~m}$, and $n=150$, a least-squares minimization between the model and the measurements, as well as Eq. 22. yield the values of $r_{\text {coil }}, r_{\text {core }}, E_{0}, S_{0}$ and $B_{V_{s}}$ in Table 1 . Figures $8 \mathrm{~b}, 8 \mathrm{c}$ show that the simulations obtained with these parameters match the measurements quite well for several input amplitudes (see also [29]).

\section{ILLUSTRATIVE EXAMPLES}

In this section, we illustrate a possible use of our ferromagnetic coil model with two examples: a band-pass filter, and a transformer. In order to specifically highlight the effect of magnetic non-linearities, both examples are kept minimal. For the band-pass filter, we consider the passive sub-circuit only, and ignore transistors and feedback. For the transformer, the input stage (typically, an amplifier) is not considered.

\subsection{Band-pass filter}

We consider the passive band-pass filter of a well-known wah-wah pedal circuit (Fig. 9a, for which the influence of the core non-linearities on the resulting sound are proeminent. 


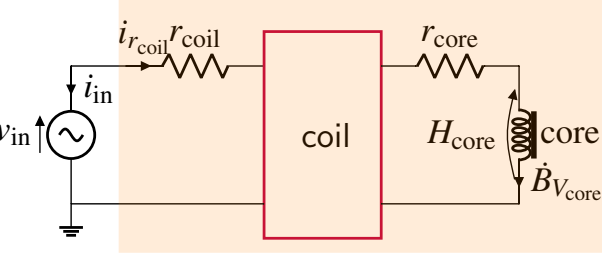

(a) Voltage-controlled ferromagnetic coil.

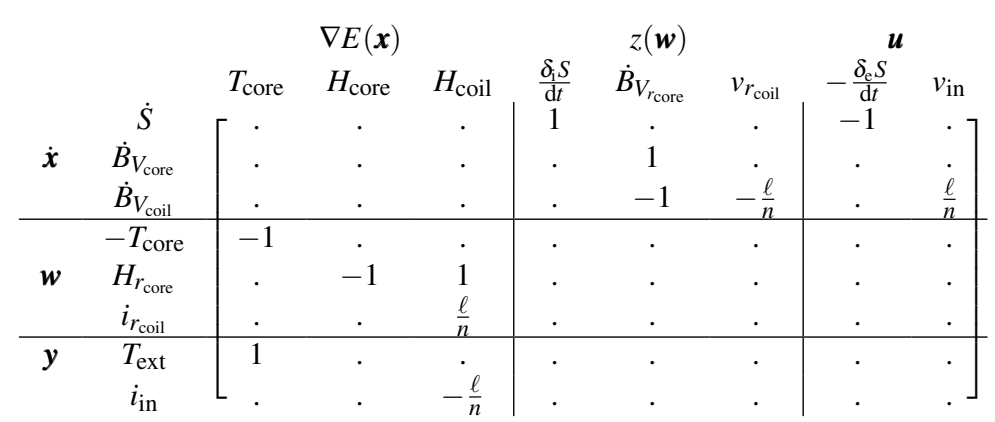

(b) Corresponding PHS. Dots represent zeros.

Figure 7: Equivalent circuit and PHS for the voltage-controlled ferromagnetic coil.

\begin{tabular}{ccccccccc}
\hline$r_{1} \& r_{2} \& r_{\text {coil }}(\Omega)$ & $r_{\text {core }}(\Omega)$ & $L_{1} \& L_{2}(\mathrm{H})$ & $R(\Omega)$ & $V R_{1}(\Omega)$ & $E_{0}(\mathrm{~J})$ & $S_{0}\left(\mathrm{~J} . \mathrm{K}^{-1}\right)$ & $B_{V_{s}}\left(\mathrm{~J} . \mathrm{A}^{-1} \cdot \mathrm{m}\right)$ & $f_{s}(\mathrm{~Hz})$ \\
\hline 15 & $3.3 e^{-6}$ & $5 e^{-3}$ & $1 \mathrm{k}$ & $5 \mathrm{k}$ & 13.09 & $4.32 e^{-2}$ & $6.61 e^{-6}$ & $192 \mathrm{k}$ \\
\hline
\end{tabular}

Table 1: Simulation parameters for the band-pass and transformer.

For this band-pass filter, the input is the $Q 2$ transistor emitter voltage, and the output is the $V R_{1}$ potentiometer voltage (Fig. 9b, where the component $L_{1}$ represents the entire ferromagnetic coil equivalent circuit). Resistors are linear and obey Ohm's law $v_{R}=R i_{R}$. Capacitors are linear, of state $q_{C}$ and energy $E_{C}\left(q_{C}\right)=q_{C}^{2} /(2 C)$. These relations, associated with Kirchhoff's laws and Eqs. (14)-(19)-(21)(22)-(25), allow a PHS formulation of the band-pass.

The complete PHS is given on Fig. 9c

A simulation is performed using a Newton-Raphson algorithm on the discretized PHS (see e.g. [29, 30] for more details on the discretization and integration), with $v_{\text {in }}=$ $U_{0} \sin \left(2 \pi f_{0} t\right)$. For the parameters given in Table 1, and component values on Fig. 9 a the center frequency is $f_{c} \approx$ $2 \mathrm{kHz}$, and the bandwith is $\Delta_{f} \approx 1 \mathrm{kHz}$. Figures $9 \mathrm{~d}$. 9f show the simulation results for different input frequencies $f_{0}$ and $U_{0}=25 \mathrm{~V}$. The core saturation and hysteresis are mostly observed for input frequencies in the filter bandwidth.

Sound examples are available at https://github. $\mathrm{com} / \mathrm{JNaj} / \mathrm{jaes}$ ferromag.

\subsection{Transformer with ferromagnetic core}

Output transformers are generally present in amplifiers, to reduce the load impedance seen by the loudspeaker [31. 2]. Transformers with a ferromagnetic core are considered particularly interesting because of the core high magnetic permeability, which reduces the leakage flux [32].

We build a non-linear transformer model by coupling two ferromagnetic coils, that is, letting them share the same core (Fig. 10a). The primary (resp. secondary) coil inductor and associated resistor are denoted $L_{1}$ and $r_{1}$ (resp. $L_{2}$ and $r_{2}$ ), with number of turns $n_{1}$ (resp. $n_{2}$ ). To simplify the interconnection matrix, we define for the magnetic domain the flow $f=\dot{B}_{V} / \ell$ and effort $e=H \ell$. Figure $10 \mathrm{~b}$ shows the corresponding circuit, with gyrators defined by:

$$
\begin{aligned}
& {\left[\begin{array}{l}
e_{1} \\
v_{1}
\end{array}\right]=\left[\begin{array}{cc}
0 & n_{1} \\
-n_{1} & 0
\end{array}\right]\left[\begin{array}{l}
f_{1} \\
i_{1}
\end{array}\right],} \\
& {\left[\begin{array}{l}
v_{2} \\
e_{2}
\end{array}\right]=\left[\begin{array}{cc}
0 & n_{2} \\
-n_{2} & 0
\end{array}\right]\left[\begin{array}{l}
i_{2} \\
f_{2}
\end{array}\right] .}
\end{aligned}
$$

Kirchhoff's laws and Eqs. (14)-(19)-(20)-(25)-(29) allow a PHS formulation of the transformer.

The complete PHS is shown on Fig. 10c

A simulation is performed (same technique as for the band-pass) with $v_{\text {in }}=U_{0} \sin \left(2 \pi f_{0} t\right)$, and parameters in Table 1. Figures 10d 10e show simulation results for different input voltages and $f_{0}=100 \mathrm{~Hz}$. It can bee seen that saturation and hysteresis arise for large input voltages, while the transformer's behavior for small input voltages is quasi-linear. This corresponds qualitatively to observations on real transformers.

It is worth noting that the modularity of the model would allow for more complex transformer topologies (multiple primaries, multiple secondaries, air-gaps ...) without difficulty.

\section{CONCLUSION}

In this paper, a new macroscopic model of ferromagnetic coil has been developed. It is based upon a componentwise, energetic approach. Characteristics like saturation and hysteresis are well reproduced, as well as the influence of thermodynamics. This explicit influence is an improvement with respect to other models. Moreover, the modularity of the model makes it particularly versatile, as the component approach can be applied to multiple combinations of cores and coils.

As an illustration, the model has been used to simulate two representative audio sub-circuits: a band-pass filter, and a transformer. Due to the reduced number of param- 


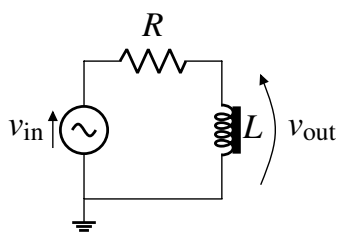

(a) Experimental setup for measurements on an inductor

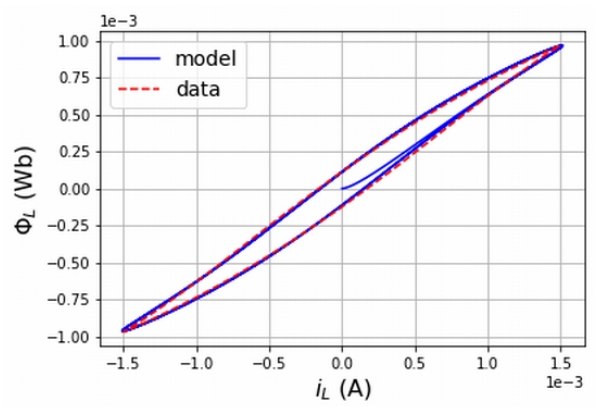

(b) Measurements versus model for a voltage input amplitude of $400 \mathrm{mV}$.

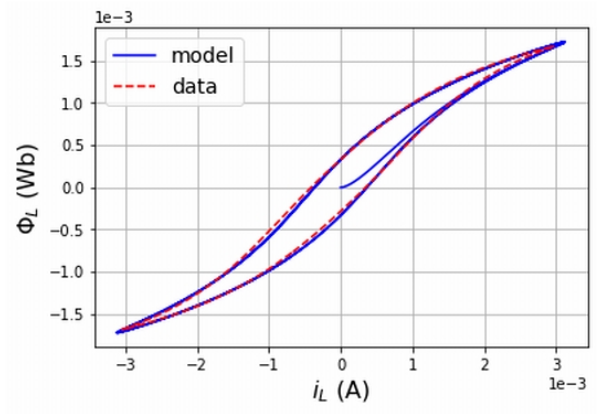

(c) Measurements versus model for a voltage input amplitude of $800 \mathrm{mV}$.

Figure 8: Experimental setup and comparison to measurements for a Fasel Red inductor.

eters and variables, these simulations can be performed in real-time.

Nonetheless, even if the model has been successfully identified with real components, and exhibits a satisfactory qualitative behavior for audio applications, extensive measurements should be conducted to assess the model relevance more quantitatively. This shall be the object of future work.

\section{Bibliography}

[1] D. C. Jiles, D. L. Atherton, "Theory of ferromagnetic hysteresis," Journal of Magnetism and Magnetic Materials, vol. 61, no. 1-2, pp. 48-60 (1986).

[2] J. Macak, J. Schimmel, "Simulation of a vacuumtube push-pull guitar power amplifier," presented at the Proceedings of the 15th International Conference on Digital Audio Effects (DAFx-12), York, United Kingdom (2012).

[3] M. Holters, U. Zölzer, "Circuit simulation with inductors and transformers based on the Jiles-Atherton model of magnetization," presented at the Proceedings of the 19th International Conference on Digital Audio Effects (DAFx-16), Brno, Czench Republic, pp. 55-60 (2016).

[4] S. E. Zirka, Y. I. Moroz, R. G. Harrison, K. Chwastek, "On physical aspects of the Jiles-Atherton hysteresis models," Journal of Applied Physics, vol. 112, no. 4, p. 043916 (2012).

[5] D. C. Hamill, "Gyrator-capacitor modeling: A better way of understanding magnetic components," presented at the Proceedings of 1994 IEEE Applied Power Electronics Conference and Exposition-ASPEC'94, pp. 326-332 (1994).

[6] Q. Chen, L. Xu, X. Ruan, S. C. Wong, C. K. M. Tse, "Gyrator-Capacitor Simulation Model of Nonlinear Magnetic Core," 2009 Twenty-Fourth Annual IEEE Applied Power Electronics Conference and Exposition, pp. 1740-1746 (2009).

[7] R. C. D. de Paiva, J. Pakarinen, V. Välimäki, M. Tikander, "Real-time audio transformer emulation for virtual tube amplifiers," EURASIP Journal on Advances in Signal Processing, vol. 2011, no. 1, p. 347645 (2011).

[8] M. Sowa, Ł. Majka, "Ferromagnetic core coil hysteresis modeling using fractional derivatives," Nonlinear Dynamics, vol. 101, no. 2, pp. 775-793 (2020).

[9] V. François-Lavet, F. Henrotte, L. Stainier, L. Noels, C. Geuzaine, "An energy-based variational model of ferromagnetic hysteresis for finite element computations," Journal of Computational and Applied Mathematics, vol. 246, pp. 243-250 (2013).

[10] G. Bertotti, "Dynamic generalization of the scalar Preisach model of hysteresis," IEEE Transactions on Magnetics, vol. 28, no. 5, pp. 2599-2601 (1992).

[11] M. LoBue, V. Basso, C. P. Sasso, G. Bertotti, "Entropy and entropy production in magnetic systems with hysteresis," Journal of Applied physics, vol. 97, no. 10, p. 10E513 (2005).

[12] B. M. Maschke, A. J. Van der Schaft, P. Breedveld, "An intrinsic Hamiltonian formulation of network dynamics: Non-standard Poisson structures and gyrators," Journal of the Franklin Institute, pp. 923-966 (1992).

[13] A. Van der Schaft, D. Jeltsema, et al., "PortHamiltonian systems theory: An introductory overview," Foundations and Trends in Systems and Control, vol. 1, no. 2-3, pp. 173-378 (2014).

[14] A. Falaize, T. Hélie, "Passive guaranteed simulation of analog audio circuits: A port-Hamiltonian approach," Applied Sciences, vol. 6, no. 10, p. 273 (2016).

[15] V. Duindam, A. Macchelli, S. Stramigioli, $\mathrm{H}$. Bruyninckx, Modeling and control of complex physical systems: the port-Hamiltonian approach (Springer Science \& Business Media) (2009).

[16] E. Ising, "Beitrag zur theorie des ferromagnetismus," Zeitschrift für Physik, vol. 31, no. 1, pp. 253258 (1925).

[17] G. F. Newell, E. W. Montroll, "On the theory of the Ising model of ferromagnetism," Reviews of Modern Physics, vol. 25, no. 2, p. 353 (1953). 


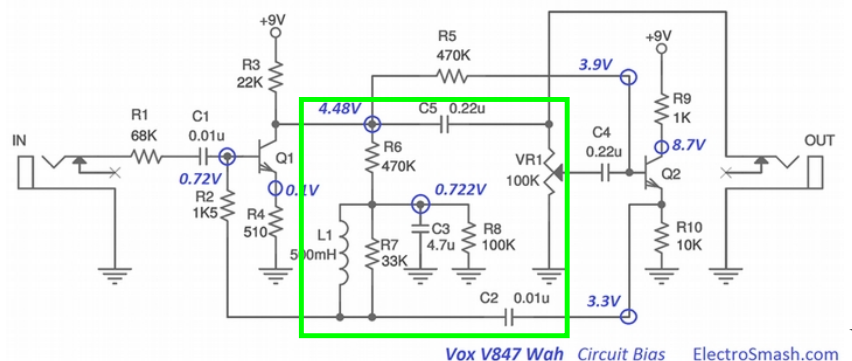

(a) Vox V847 circuit (from ElectroSmash https://www. electrosmash.com/vox-v847-analysis) and passive sub-circuit framed in green.

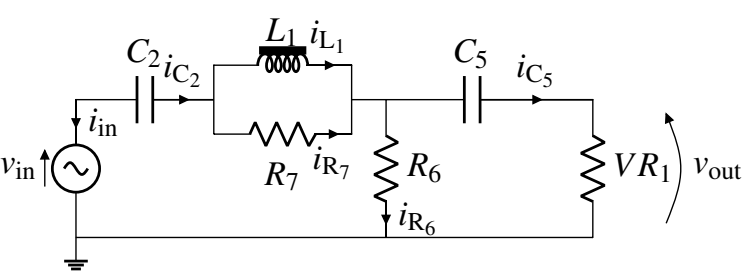

(b) Simplified passive band-pass filter of the Vox V847 circuit.

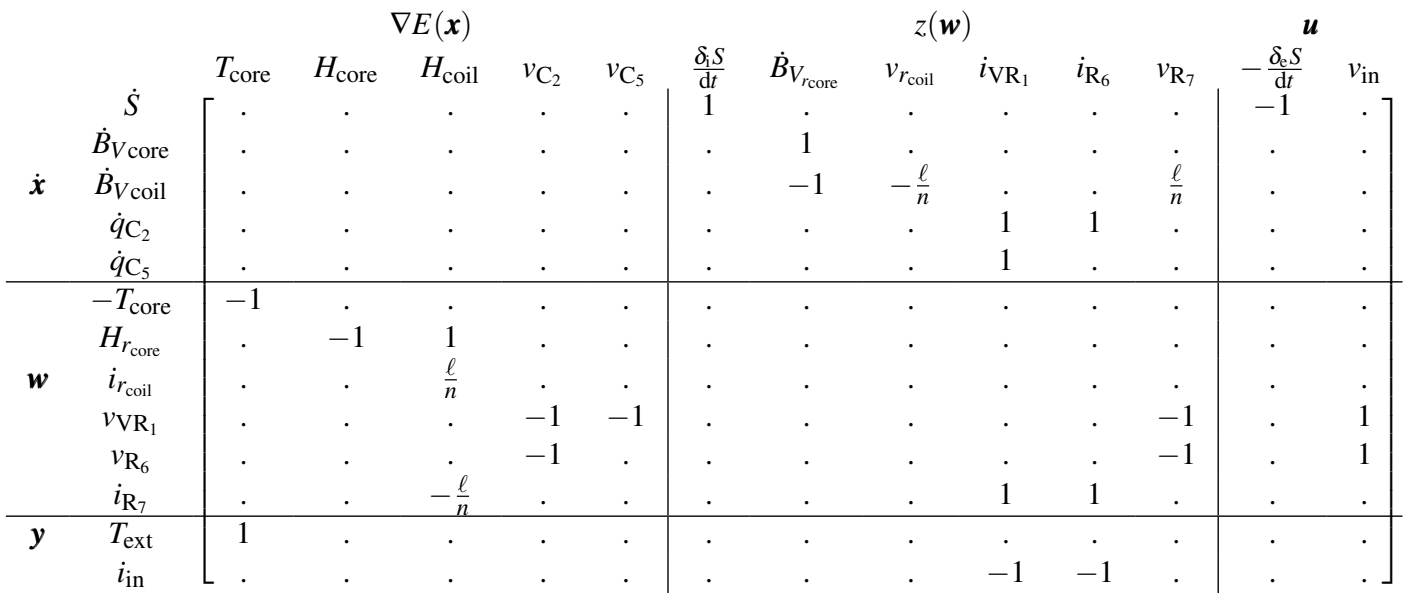

(c) Corresponding PHS. Dots represent zeros.

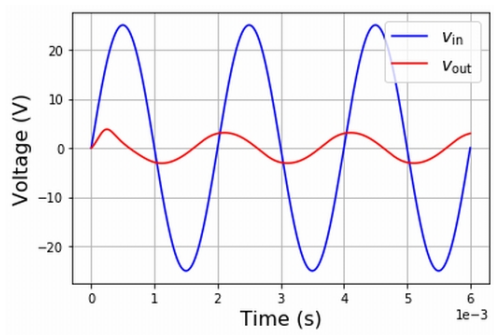

(d) Simulation for $f_{0}=500 \mathrm{~Hz}$.

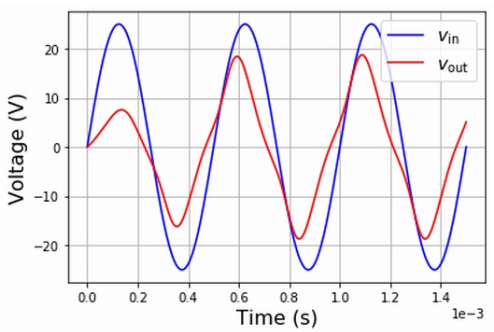

(e) Simulation for $f_{0}=2000 \mathrm{~Hz}$.

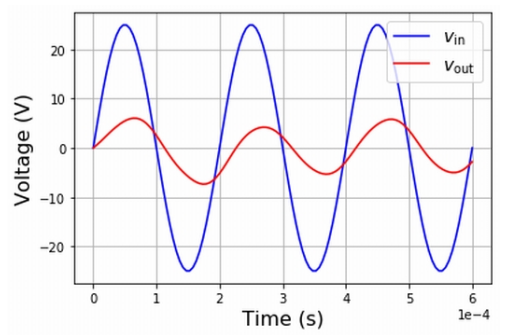

(f) Simulation for $f_{0}=5000 \mathrm{~Hz}$.

Figure 9: Circuit, PHS and simulation results for the passive band-pass filter.

[18] J. Strecka, M. Jascur, "A brief account of the Ising and Ising-like models: Mean-field, effective-field and exact results," arXiv preprint arXiv:1511.03031 (2015).

[19] T. L. Hill, An introduction to statistical thermodynamics (Courier Corporation) (1986).

[20] H. B. Callen, "Thermodynamics and an Introduction to Thermostatistics," (1998).

[21] P. T. Landsberg, Thermodynamics and statistical mechanics (Courier Corporation) (2014).

[22] A. Liechtenstein, M. Katsnelson, V. Gubanov, "Exchange interactions and spin-wave stiffness in ferromagnetic metals," Journal of Physics F: Metal Physics, vol. 14, no. 7, p. L125 (1984).

[23] E. T. Jaynes, "On the rationale of maximumentropy methods," Proceedings of the IEEE, vol. 70, no. 9, pp. 939-952 (1982).
[24] K. Stowe, An introduction to thermodynamics and statistical mechanics (Cambridge University Press) (2007).

[25] F. Utermohlen, "Mean Field Theory Solution of the Ising Model," https://cpb-us-w2.wpmucdn. com/u.osu.edu/dist/3/67057/files/2018/ 09/Ising_model_MFT-25b1klj.pdf.

[26] G. Bertotti, Hysteresis in magnetism: for physicists, materials scientists, and engineers (Academic press) (1998).

[27] L. O. Chua, C. A. Desoer, E. S. Kuh, Linear and nonlinear circuits (McGraw-Hill) (1987).

[28] C. Kittel, "Physical theory of ferromagnetic domains," Reviews of modern Physics, vol. 21, no. 4, p. 541 (1949).

[29] J. Najnudel, R. Müller, T. Hélie, D. Roze, "A power-balanced dynamic model of ferromagnetic coils," 


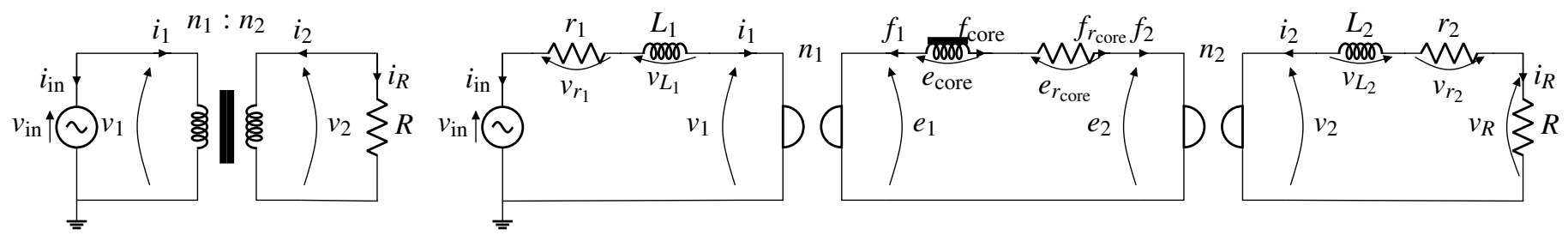

(a) Transformer loaded with a resistor.

(b) Equivalent gyrator-capacitor representation.

\begin{tabular}{|c|c|c|c|c|c|c|c|c|c|c|c|c|}
\hline & & & $\nabla E$ & & & & & $(\boldsymbol{w})$ & & & t & \\
\hline & & $T_{\text {core }}$ & $e_{\text {core }}$ & $i_{L_{1}}$ & $i_{L_{2}}$ & $\frac{\delta_{\mathrm{i}} S}{\mathrm{~d} t}$ & $f_{r_{\text {core }}}$ & $v_{r_{1}}$ & $v_{r_{2}}$ & $v_{R}$ & $-\frac{\delta_{\mathrm{e}} S}{\mathrm{~d} t}$ & $v_{\text {in }}$ \\
\hline & $\dot{S}$ & [ · & . & . & . & 1 & . & . & . & . & -1 & $\cdot]$ \\
\hline$\dot{x}$ & $f_{\text {core }}$ & . & . & . & . & . & 1 & . & . & . & . & . \\
\hline & $\dot{\Phi}_{L_{1}}$ & . & . & . & . & . & $-n_{1}$ & -1 & . & . & . & 1 \\
\hline & $\dot{\Phi}_{L_{2}}$ & . & . & . & . & . & $-n_{2}$ & . & -1 & 1 & . & . \\
\hline & $-T_{\text {core }}$ & -1 & . & . & . & . & . & . & . & . & . & . \\
\hline & $e_{r_{\text {core }}}$ & . & -1 & $n_{1}$ & $n_{2}$ & . & . & . & . & . & . & . \\
\hline$w$ & $i_{r_{1}}$ & . & . & 1 & . & . & . & . & . & . & . & . \\
\hline & $i_{r_{2}}$ & . & . & . & 1 & . & . & . & . & . & . & . \\
\hline & $i_{R}$ & . & . & . & -1 & . & . & . & . & . & . & . \\
\hline$y$ & $T_{\text {ext }}$ & 1 & . & $\cdot$ & . & . & . & . & . & . & . & . \\
\hline & $i_{\text {in }}$ & L . & . & -1 & . & . & . & . & . & . & . & \\
\hline
\end{tabular}

(c) Corresponding PHS.

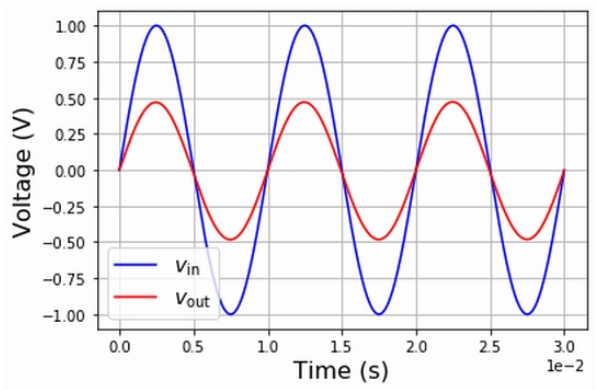

(d) Simulation for $U_{0}=1 \mathrm{~V}$, $n_{1} / n_{2}=2$.

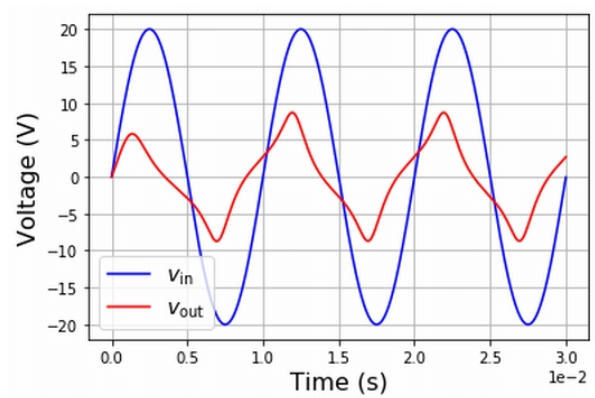

(e) Simulation for $U_{0}=20 \mathrm{~V}$, $n_{1} / n_{2}=2$.

Figure 10: Circuit, PHS and simulation results for the transformer.

presented at the 23rd International Conference on Digital Audio Effects (eDAFx-20) (2020).

[30] J. Najnudel, T. Hélie, D. Roze, H. Boutin, "Simulation of an ondes Martenot circuit," IEEE/ACM Transactions on Audio, Speech, and Language Processing, vol. 28, pp. 2651-2660 (2020).

[31] I. Cohen, T. Hélie, "Real-time simulation of a guitar power amplifier," presented at the Proceedings of the 13th International Conference on Digital Audio Effects (DAFx-10), Graz, Austria (2010).

[32] C. W. T. McLyman, Transformer and inductor design handbook (CRC press) (2016).

\section{APPENDIX}

\section{A.1 Energy derivative w.r.t the entropy}

From Eq. [14, we deduce:

$$
\begin{aligned}
\frac{\partial E_{\text {core }}}{\partial S}\left(\boldsymbol{x}_{\text {core }}\right) & \stackrel{1}{=}-\frac{E_{0}}{S_{0}}\left|\frac{B_{V_{\text {core }}}}{B_{V_{s}}}\right| \frac{f^{-1^{\prime}}\left(\frac{S}{S_{0}}\right)}{\cosh ^{2}\left(f^{-1}\left(\frac{S}{S_{0}}\right)\right)} \\
& \stackrel{2}{=} \frac{E_{0}}{S_{0}}\left|\frac{B_{V_{\text {core }}}}{B_{V_{s}}}\right| \frac{\cosh ^{2}\left(f^{-1}\left(\frac{S}{S_{0}}\right)\right)}{\cosh ^{2}\left(f^{-1}\left(\frac{S}{S_{0}}\right)\right) f^{-1}\left(\frac{S}{S_{0}}\right)} \\
& \stackrel{3}{=} \frac{E_{0}}{S_{0}}\left|\frac{B_{V_{\text {core }}}}{B_{V_{s}}}\right| \frac{1}{f^{-1}\left(\frac{S}{S_{0}}\right)} \stackrel{4}{=} T
\end{aligned}
$$

using 1. $\tanh ^{\prime} u=u^{\prime} / \cosh ^{2} u, 2 . f^{-1^{\prime}}=1 /\left(f^{\prime} \circ f^{-1}\right)$ and $f^{\prime}(u)=-u / \cosh ^{2}(u), 4 . E_{0} / S_{0}=T_{c}$ and $f^{-1}\left(S / S_{0}\right)=$ 
$\left|B_{V_{\text {core }}} / B_{V_{s}}\right| T_{c} / T$, where the symbol $\circ$ stands for function composition.

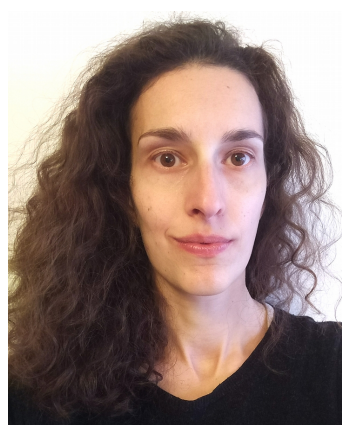

Judy Najnudel

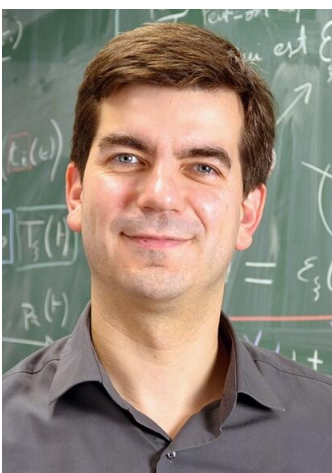

Thomas Hélie
Judy Najnudel is graduated from École Nationale Supérieure Louis Lumière (Master in 2007) and Sorbonne Université (ATIAM MSc in 2018). She is currently pursuing a $\mathrm{PhD}$ at STMS laboratory (Sciences and Technologies for Music and Sound, IRCAM-CNRS-SU). She focuses on physical modeling of nonlinear analog circuits for audio applications.

Thomas Hélie is graduated from the École Nationale Supérieure des Télécommunications de Bretagne (Ing. Dipl. in 1997), Université Paris VI (ATIAM MSc in 1998 and « Habilitation à diriger des Recherches » in 2013) and Université Paris XI (MSc ATS in 1998 and PhD in 2002). Since 2017, he is director of research at CNRS, head of the S3AM team at STMS laboratory and coordinator of the ATIAM MSc. His research topics include nonlinear dynamical system and control theory, signal processing, acoustics, and physical modeling of audio and musical instruments

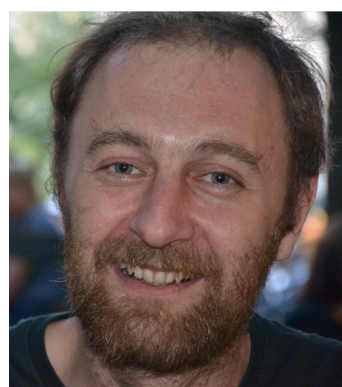

David Roze

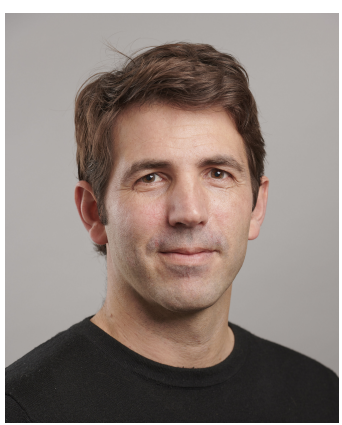

Rémy Müller
David Roze is graduated from INSA de Rouen (Ing. Dipl. in 2005) and Université Paris VI (ATIAM MSc in 2006 and $\mathrm{PhD}$ in 2010). He is a CNRS researcher at STMS laboratory. His research interests include control of nonlinear dynamical systems, sound synthesis, and numerical methods for real-time computation.

Rémy Müller is graduated from Institut National des Sciences Appliquées (Ing. Dipl. in 2003) and Université Lyon I (Images and Systems MSc in 2004). He also attended Université Paris VI as a free listener (ATIAM Msc in 2004). He has been working for 13 years as an audio signal processing engineer, he is a part-time $\mathrm{PhD}$ student sharing his time between the S3AM team at STMS laboratory, and the UVI company, Paris, France. His current research interests include the physical modeling of non-linear dynamical systems using port-Hamiltonian Systems, powerbalanced numerical integration methods, non-bandlimited signal processing and reverberation. 